\title{
Anisotropy in multi-phase systems: a phase field approach
}

\author{
HARALD GARCKE ${ }^{\dagger}$, BARBARA STOTH ${ }^{\ddagger}$ \\ Institut für Angewandte Mathematik, Wegelerstr. 6, D-53115 Bonn, Germany \\ AND \\ BRITTA NESTLER ${ }^{\S}$ \\ Foundry Institute, RWTH Aachen, Intzestr. 5, D-52072 Aachen, Germany
}

[Received 11 September 1998 and in revised form 7 May 1999]

\begin{abstract}
A phase field concept for interface motion in general multi-phase systems with anisotropic interfacial energy is studied. We allow the anisotropy to be even crystalline which leads to a polygonial Wulff shape. A sharp interface model which appears as the limit of small interfacial thickness is stated.

Through a series of numerical simulations we demonstrate that our concept can recover features like crystalline curvature flow, an anisotropic version of Young's law at triple-junctions and an anisotropic modification of the right angle condition at points where the interface intersects an external boundary. An important advantage of our approach is that there is a simple relation between the coefficients in the phase field model and the defining parameters of the sharp interface model.
\end{abstract}

\section{Introduction}

In this paper, we study, how it is possible to describe the evolution in multi-phase systems using a multi-phase-field concept. We are interested in multi-phase systems that aim to decrease an anisotropic, may be even crystalline, surface energy. In such systems the interface between different phases can be modelled either by a hypersurface or by a diffuse interface. In the latter case, one describes the interface by an interfacial layer with a small but finite thickness. Some examples of such systems are the various phase field models. The multi-phase-field model is based on a vector of order parameters. The order parameters can represent either different phases in multiphase systems or different orientational variants in a polycrystalline microstructure. With these two interpretations for the order parameters, the phase field concept can be used to model phenomena like phase transformations and grain boundary motion in a rather general setting. This will include multi-phase evolution with anisotropic surface energies which are allowed to be crystalline.

The simplest phase field (or order parameter) model is the Allen-Cahn equation which was introduced to model the evolution of antiphase boundaries. First by formal asymptotic expansions [23] and later rigorously [7], it was shown that the Allen-Cahn equation in the limit, as the interfacial thickness tends to zero, leads to interfaces evolving by mean curvature flow. By now there are a number of results on the relationship between phase field (or order parameter) models and sharp interface models in the two phase case. For example, it is known that a phase field equation coupled

\footnotetext{
†Email: harald@iam.uni-bonn.de

†Email: bstoth@iam.uni-bonn.de

§Email: b.nestler@gi.rwth-aachen.de
} 
to an energy balance leads to the modified Stefan problem with Gibbs-Thomson law and kinetic undercooling $[25,28]$.

Kobayashi [19] observed in numerical simulations that, in order to describe complicated morphologies like dendritic growth, it is necessary to incorporate anisotropy into the system. He proposed a phase field model which included anisotropy effects and, by now, it is verified that one recovers anisotropic versions of sharp interface models in the small interfacial thickness limit (see $[10,21])$.

The main new feature in multi-phase situations is the appearance of junctions of three and more phases. Bronsard \& Reitich [4] were the first to show by formal asymptotic expansions that a balance of forces at multiple-junctions known as Young's law can be recovered in the sharp interface limit of an Allen-Cahn system. By now, there are also generalizations of this result to anisotropic situations in which torque terms enter the balance law at multiple-junctions (see [13, 22, 33]).

In the present paper, we study a phase field model which is based on a generalized GinzburgLandau free energy

$$
\mathcal{E}(\mathbf{u})=\int_{\Omega}\left(\varepsilon f(\mathbf{u}, \nabla \mathbf{u})+\frac{1}{\varepsilon} \Psi(\mathbf{u})+\Phi(\mathbf{u})\right)(x) \mathrm{d} x .
$$

This energy functional is defined for a multi-phase-field vector $\mathbf{u}=\left(u_{1}, \ldots, u_{N}\right): \Omega \rightarrow \mathbb{R}^{N}$, $N \in \mathbb{N}$, where $\Omega \subset \mathbb{R}^{d}, d \in \mathbb{N}$, is an open and bounded set. Here, $f$ is a generalized gradient free energy and $\Psi$ and $\Phi$ are bulk potentials. The potential $\Psi$ is assumed to have global minima which correspond to the phases or grains, respectively, of the system and $\Phi$ accounts for deviations from thermodynamical equilibrium. In the following sections, we will use an ansatz for the gradient part of the free energy which is expressed in terms of the irreducible representations $u_{i} \nabla u_{j}-u_{j} \nabla u_{i}$. This idea was introduced in the isotropic case by Steinbach et al. [26] and anisotropy was included into the model by Garcke, Nestler \& Stoth [13]. Since we want to concentrate on interfacial effects, we will not incorporate any bulk diffusion into our consideration. In this case, the phase field model will be simply the $L^{2}$-gradient flow of the free energy $\mathcal{E}$.

In our previous work [13], we demonstrated how anisotropy in the energy $\mathcal{E}$ leads to the anisotropic mean curvature flow along the interface, to the anisotropic form of Young's law at multiple-junctions and to a correction of the $90^{\circ}$ angle condition at intersections of interfaces with the external boundary which is due to shear forces. In the isotropic case, only a tangential force trying to contract the interface is present. In anisotropic situations also a shear force tending to rotate the surface towards directions of lower energy acts on the interface (see [16] and [17]). It is the goal of this paper to show via numerical simulations that the phase field model is able to recover the influence of these forces on the behaviour of the interfaces.

In Section 2, we state the phase field concept of $[13,26]$ and describe how it is related to sharp interface models. We also show how specific anisotropies can be incorporated into the concept. Two qualitatively different choices of anisotropy are introduced. One is a smooth anisotropy with a surface energy which is differentiable. A second one is a crystalline anisotropy with a surface energy which is non-differentiable. For a crystalline surface energy the Wulff shape is polyhedral (see [15]). The crystalline phase field approach to approximate sharp interface motion is justified, so far, only in the two phase case with constant driving forces (see [2]). It is important to understand the crystalline motion in the phase field setting, because there are many relevant applications as, for example, grain growth, crystalline dendritic growth, faceted eutectic and peritectic phase transformations for which it would be desirable to have a phase field model. We remark that in many applications an anisotropic phase field model for more than two order parameters is necessary. 
The main part of this paper, Section 3, consists of a description of numerical simulations using the phase field approach. In particular, our goal is to demonstrate that it is possible to use the phase field methodology also in the anisotropic multi-phase case. We note that the free energy, as introduced in [13,14], gives enough freedom to choose different anisotropic free energies for interfaces separating pairs of phases $i$ and $j(i, j=1, \ldots, N)$. When using a phase field approximation to a sharp interface problem, one requirement is that the parameters in the free energy $\mathcal{E}$ can be easily related to the parameters which define the sharp interface model. In our study [14], we demonstrated that a variant of a multi-obstacle potential has the best behaviour with respect to this requirement. Therefore, we used it in our simulations.

In the case of smooth anisotropies, we compare results of the numerical simulations with our asymptotic analysis [13]. Here, in particular the role of the Herring torque terms which appear in the anisotropic setting is considered at points where an interface meets the external boundary and at triple-junctions.

As pointed out before, in the crystalline multi-phase case it is less clear what happens. But our numerical simulations give a high agreement to the theoretical predictions in the two phase case (see also the recent work of Belletini, Goglione \& Novaga [2]). As one application of our approach we consider the evolution of grain boundary trijunctions in epitaxial growth. Thangaraj, Westmacott and Dahmen [31,32] observed that in thin films of $\mathrm{Al}$ on a Si substrate two families of trijunctions with different symmetry occur. The possible trijunctions were classified by Cahn \& Kalonji [6] and Cahn posed the question whether it is possible to model this kind of phenomenon with a phase field model. We demonstrate in Section 3 that this is possible with our approach and we will present more details in a forthcoming paper [12].

\section{The Phase Field Model}

\subsection{The general concept}

We briefly describe the multi-phase-field model as introduced by Steinbach et al. [26] and Garcke, Nestler \& Stoth [13]. For simplicity, we restrict ourselves to two space dimensions. Generalizations to higher space dimensions are possible.

To model interface motion in a system of $N$ phases or grains, we introduce a vector of order parameters $\mathbf{u}=\left(u_{1}, \ldots, u_{N}\right)$, where $u_{i}$ describes the fraction of phase $i$ in a multi-phase system of the fraction of the orientational variant labelled $i$ in a polycrystalline structure. The phase space for such a multi-order parameter $\mathbf{u}$ is defined as

$$
\Sigma:=\left\{\mathbf{u} \in \mathbb{R}^{N}: \sum_{i=1}^{N} u_{i}=1\right\} .
$$

In view of this constraint, we define the projection $T$ onto the tangent plane $T \Sigma:=\left\{\mathbf{u} \in \mathbb{R}^{N}\right.$ : $\left.\sum_{i=1}^{N} u_{i}=0\right\}$, by

$$
(T \mathbf{u})_{i}:=u_{i}-\frac{1}{N} \sum_{j=1}^{N} u_{j} .
$$

Since $u_{i}$ stands for a fraction, we ask all components of the order parameter $\mathbf{u}$ to be non-negative, i.e. we want $\mathbf{u} \in \mathcal{G}$, where

$$
\mathcal{G}:=\left\{\mathbf{u} \in \Sigma: u_{i} \geqslant 0\right\}
$$


is the Gibbs simplex in $\mathbb{R}^{N}$.

We now consider the general vectorial Allen-Cahn system for a multi-order parameter

$$
\mathbf{u}:(0, T) \times \Omega \rightarrow \Sigma,
$$

on an open, bounded and piecewise smooth domain $\Omega \subset \mathbb{R}^{2}$. It takes the form

$$
\varepsilon \beta(\mathbf{u}, \nabla \mathbf{u}) \mathbf{u}_{t}=\varepsilon \operatorname{div} T f_{, X}(\mathbf{u}, \nabla \mathbf{u})-\varepsilon T f_{, \mathbf{u}}(\mathbf{u}, \nabla \mathbf{u})-\frac{1}{\varepsilon} T \Psi_{, \mathbf{u}}(\mathbf{u})-T \Phi_{, \mathbf{u}}(\mathbf{u}),
$$

together with the natural boundary condition

$$
T f_{, X}(\mathbf{u}, \nabla \mathbf{u}) \cdot v_{\partial \Omega}=0 \quad \text { on } \quad \partial \Omega,
$$

where $v_{\partial \Omega}$ is the outer unit normal to $\partial \Omega$. Here, $\varepsilon$ is a generally small length scale parameter. The anisotropic kinetic coefficient $\beta$ is assumed to be strictly positive and homogeneous of degree zero in the gradient variable. The functions $f$ and $\Psi$ model surface energy and bulk energy, respectively and $\Phi$ models deviations from thermodynamical equilibrium. We assume that $f$ and $\Psi$ are nonnegative and

$$
\Psi(\mathbf{u})=0 \text { for } \mathbf{u} \in \Sigma \quad \Leftrightarrow \quad \mathbf{u}=\mathbf{e}^{i},
$$

where $\mathbf{e}^{i}$ denotes the $i$ th standard unit vector in $\mathbb{R}^{N}$. In addition, we impose that $f$ is homogeneous of degree two in the gradient variable. The notation $f_{\text {, }}$ denotes the derivative with respect to $\mathbf{u}$, and $f_{, X}$ denotes the derivative with respect to the gradient variable $\nabla \mathbf{u}$.

\subsection{Relation to a sharp interface model}

As the parameter $\varepsilon$ gets small, this system aims to approximate the following sharp interface model where the interface is modelled by a curve $\Gamma$ which evolves in time. For a derivation of this asymptotic limit from the anisotropic vectorial Allen-Cahn model in the smooth case, we refer to our previous work [13].

The governing equation for an evolving interface $\Gamma$, separating bulk phases $i$ and $j$, is given by the kinetic Gibbs-Thomson law

$$
\hat{\mu}_{i j}(\theta) V=-\left(\hat{\sigma}_{i j}(\theta)+\hat{\sigma}_{i j}^{\prime \prime}(\theta)\right) \kappa+\Phi\left(\mathbf{e}^{j}\right)-\Phi\left(\mathbf{e}^{i}\right) .
$$

Here, $V$ and $\kappa$ are the normal velocity and the curvature of the interface $\Gamma . \theta$ is the angle that the normal $v$ which points into phase $j$ forms with the $x$-axis. The coefficients $\hat{\mu}_{i j}$ and $\hat{\sigma}_{i j}$ are the mobility and the surface energy associated with a transition from phase $i$ to $j$. In the anisotropic case, both the mobility and the surface energy depend on the orientation of the interface. We always assume $\hat{\sigma}_{i j}(\theta)=\hat{\sigma}_{j i}(\theta+\pi)$.

At triple-junctions $m$, where three interfaces $\Gamma_{1}, \Gamma_{2}$ and $\Gamma_{3}$, intersect, Young's law

$$
\left(\hat{\sigma}_{i j}\left(\theta_{1}\right) v_{1}+\hat{\sigma}_{i j}^{\prime}\left(\theta_{1}\right) \tau_{1}\right)+\left(\hat{\sigma}_{j k}\left(\theta_{2}\right) \nu_{2}+\hat{\sigma}_{j k}^{\prime}\left(\theta_{2}\right) \tau_{2}\right)+\left(\hat{\sigma}_{k i}\left(\theta_{3}\right) \nu_{3}+\hat{\sigma}_{k i}^{\prime}\left(\theta_{3}\right) \tau_{3}\right)=0
$$

has to be satisfied. Here, $\tau_{i}$ is the tangent to $\Gamma_{i}$, so that $\left(v_{i}, \tau_{i}\right)$ form a positively orientated basis of $\mathbb{R}^{2}$. At boundary points $m \in \partial \Omega$, where an interface $\Gamma$ and the domain boundary intersect, the natural boundary condition

$$
\left(\hat{\sigma}_{i j}(\theta) v+\hat{\sigma}_{i j}^{\prime}(\theta) \tau\right) \cdot v_{\partial \Omega}=0,
$$


has to hold, whenever $v_{\partial \Omega}$ exists at $m \in \partial \Omega$.

In [13] we showed that the surface energies $\sigma_{i j}(v)$ can be determined from the energies $f$ and $\Psi$ of the Allen-Cahn model by evaluating the energy of a stationary wave solution $\mathbf{q}$ which is orientated in direction $v$ and which connects the two minima $\mathbf{e}^{i}$ and $\mathbf{e}^{j}$ of $\Psi$. The expression we derived generalizes a formula which was in the isotropic case first obtained by Sternberg [27] and reads as

$$
\sigma_{i j}(v)=2 \int_{-\infty}^{\infty} \sqrt{\Psi(\mathbf{q}) f\left(\mathbf{q}, \mathbf{q}^{\prime} \otimes v\right)}(z) \mathrm{d} z
$$

The mobility $\mu_{i j}$ can be computed to be

$$
\mu_{i j}(v)=\int_{-\infty}^{\infty} \beta\left(\mathbf{q}, \mathbf{q}^{\prime} \otimes v\right)\left|\mathbf{q}^{\prime}\right|^{2}(z) \mathrm{d} z .
$$

The quantities $\hat{\sigma}$ and $\hat{\mu}$ relate to $\sigma$ and $\mu$, respectively, via

$$
\hat{\sigma}(\theta)=\sigma(v(\theta)), \quad \hat{\mu}(\theta)=\mu(v(\theta)),
$$

with $v(\theta)=(\cos \theta, \sin \theta)$. We point out that the relation between $\sigma_{i j}, \mu_{i j}$ and $\Psi, f$ is in general non-trivial because the stationary wave solution $\mathbf{q}$ has to be determined. As it is well known for two order parameters the surface energy and the mobility are given by a simple expression in terms of $\Psi$ and $f$ (see for example [13]). For multi-order parameter systems it is in general not possible to find a simple expression.

\subsection{Free energies and anisotropy}

For $\Psi$, we take the obstacle potential (see $[3,9,14]$ )

$$
\Psi_{o b}(\mathbf{u}):=\frac{16}{\pi^{2}} \sum_{i<j} \tilde{\mu}_{i j} \tilde{\sigma}_{i j} u_{i} u_{j}, \quad \text { with } \quad \tilde{\sigma}_{i j}, \tilde{\mu}_{i j} \in(0, \infty),
$$

for $\mathbf{u} \in \mathcal{G}, \Psi_{o b}(\mathbf{u})=+\infty$, whenever $\mathbf{u} \notin \mathcal{G}$. The surface energy and mobility parameters $\tilde{\sigma}_{i j}$ and $\tilde{\mu}_{i j}$ are assumed to obey the symmetry relations $\tilde{\sigma}_{i j}=\tilde{\sigma}_{j i}$ and $\tilde{\mu}_{i j}=\tilde{\mu}_{j i}$. The obstacle potential (2.9) was first studied by Elliott \& Luckhaus [9] in the context of phase separation of multicomponent mixtures. We remark that the obstacle potential is the deep quench limit of a logarithmic type free energy given by the mean-field theory of multi-component systems (see [9]). In systems with $N \geqslant 3$ phases or orientational variants, we remark that with this choice of the potential a stationary wave connecting two minima of $\Psi_{o b}$ may not necessarily be restricted to the edges of the Gibbs simplex. As a result, third phases may be present in a transition region connecting phase $i$ and $j$ or connecting grain $i$ and $j$ (see also Kikuchi \& Cahn [18] for wetting transitions). If one wants to avoid such effects, one needs to increase the energy in the interior of the Gibbs simplex. This can be done by introducing higher order variants of the obstacle potential (see [14])

$$
\hat{\Psi}_{o b}(\mathbf{u}):=\Psi_{o b}(\mathbf{u})+\sum_{i, j, k} \sigma_{i j k} u_{i} u_{j} u_{k} .
$$

A suitable choice of the parameters $\sigma_{i j k}$ ensures that the actual minimizers connecting two minima of the potential $\hat{\Psi}_{o b}(\mathbf{u})$ lie on the boundary of the Gibbs simplex. Herewith, the interfacial region is 
localized, so that in principle, the phase-field equations need to be solved only in the neighbourhood of the interfaces. Simultaneously, a good calibration for the physical parameters $\tilde{\sigma}_{i j}$ and $\tilde{\mu}_{i j}$ can be achieved by choosing values for $\tilde{\sigma}_{i j}$ appropriately (see [14]).

The potentials $\Psi_{o b}$ and $\hat{\Psi}_{o b}$ are obstacle potentials, because they prevent the solution from lying outside the Gibbs simplex $\mathcal{G}$. This means that the boundary of the Gibbs simplex acts as an obstacle to the solution. Since we are going to use the potential $\hat{\Psi}_{o b}$, we have to solve a parabolic variational inequality instead of the Allen-Cahn equation (2.2). A solution $\mathbf{u}$ has to satisfy

$$
\begin{aligned}
\left(\varepsilon \beta(\mathbf{u}, \nabla \mathbf{u}) \mathbf{u}_{t}\right. & \left.+\varepsilon T f_{, \mathbf{u}}(\mathbf{u}, \nabla \mathbf{u})+\frac{1}{\varepsilon} T \Psi_{, \mathbf{u}}(\mathbf{u})+T \Phi_{, \mathbf{u}}(\mathbf{u}), \mathbf{v}-\mathbf{u}\right)_{L^{2}} \\
& +\left(\varepsilon T f_{, X}(\mathbf{u}, \nabla \mathbf{u}), \nabla(\mathbf{v}-\mathbf{u})\right)_{L^{2}} \geqslant 0,
\end{aligned}
$$

for all $\mathbf{v}:(0, T) \times \Omega \rightarrow \mathcal{G}$ sufficiently smooth. By $(.,)_{L^{2}}$ we denote the usual $L^{2}$-scalar product. Existence of a viscosity solution for the scalar case of such an equation with a non-constant $\beta$ is proved in Elliott \& Schätzle [11].

For the lower order bulk free energy $\Phi$, we choose

$$
\Phi_{o b}(\mathbf{u}):=\sum_{i} m_{i} u_{i}
$$

where $m_{i} \in \mathbb{R}$ stands for the bulk energy of phase $i$ in a multi-phase system. We remark that the $m_{i}$ 's are the same in the case of a multi grain system due to the fact that grain boundaries separate domains of the same phase that are misorientated to each other. To include anisotropy in the system, we set $f$ to be an anisotropic gradient potential

$$
f=f_{\text {aniso }}(\mathbf{u}, \nabla \mathbf{u}):=\sum_{i<j} \frac{\tilde{\sigma}_{i j}}{\tilde{\mu}_{i j}} A_{i j}^{2}\left(u_{i} \nabla u_{j}-u_{j} \nabla u_{i}\right)\left|u_{i} \nabla u_{j}-u_{j} \nabla u_{i}\right|^{2},
$$

where the anisotropy is written with the help of the irreducible representations $u_{i} \nabla u_{j}-u_{j} \nabla u_{i}$. Here, $A_{i j}$ are assumed to be homogeneous of degree zero. On a phase boundary between phases $i$ and $j$, the unit normal pointing into phase $j$ can be approximated by $\left(u_{i} \nabla u_{j}-u_{j} \nabla u_{i}\right) /\left|u_{i} \nabla u_{j}-u_{j} \nabla u_{i}\right|$. This motivates our choice of the anisotropic gradient energy. We also choose the time relaxation parameter in an anisotropic form

$$
\beta(u, \nabla u)=\beta_{0}+\sum_{i<j} B_{i j}\left(u_{i} \nabla u_{j}-u_{j} \nabla u_{i}\right), \quad \text { with } \quad \beta_{0} \in(0, \infty),
$$

and with $B_{i j}$ homogeneous of degree zero. We always assume $A_{i j}(y)=A_{j i}(-y)$ and $B_{i j}(y)=$ $B_{j i}(-y)$.

Specific anisotropies will now be modelled by suitable choices of the coefficients $A_{i j}$ and $B_{i j}$.

- Smooth anisotropies

As smooth anisotropies, we use a natural extension of an expression for a solid/liquid phase system by Kobayashi [19], namely

$$
A_{i j}^{s m}(y)=1+\delta_{i j} \cos \left(M_{i j}\left(\theta-\alpha_{i j}\right)\right),
$$


where $\theta=\arg (y)$ is the angle which the vector $y$ forms with the $x$-axis. Here, $M_{i j} \in \mathbb{N}$ realizes an $M_{i j}$-fold anisotropy, $\alpha_{i j} \in[0,2 \pi)$ describes the relative orientation of the anisotropy with respect to the $\mathrm{x}$-axis and $\delta_{i j} \in(-1,1)$ measures the strength of anisotropy. In addition, we assume that $\delta_{i j}=\delta_{j i}, M_{i j}=M_{j i}$ and $\alpha_{i j}=\alpha_{j i} \pm \pi$. Similarly, we take $B_{i j}^{s m}$ to be

$$
B_{i j}^{s m}(y)=\beta_{i j} \cos \left(L_{i j}\left(\theta-\gamma_{i j}\right)\right),
$$

where $\beta_{i j} \in\left(-\beta_{0}, \beta_{0}\right), \gamma_{i j} \in[0,2 \pi), L_{i j} \in \mathbb{N}$ and we assume the same symmetry relations in $i$ and $j$ as for the gradient potential. If $y=0$, then we set $B_{i j}^{s m}=0$.

The related sharp interface model is given below. The surface potential $f_{\text {aniso }}$ and the bulk potential $\Psi$ were chosen in such a way that the energy of the two phase stationary wave solution connecting $\mathbf{e}^{i}$ and $\mathbf{e}^{j}$ in direction of the unit normal $\nu$ is exactly (see $[13,14]$ )

$$
\sigma_{i j}(v)=\tilde{\sigma}_{i j} A_{i j}^{s m}(v)
$$

and therefore,

$$
\hat{\sigma}_{i j}(\theta)=\tilde{\sigma}_{i j}\left(1+\delta_{i j} \cos \left(M_{i j}\left(\theta-\alpha_{i j}\right)\right)\right) .
$$

We remark that $\hat{\sigma}_{i j}$ leads to a stable surface energy, if

$$
\left|\delta_{i j}\right| \leqslant \frac{1}{M_{i j}^{2}-1}
$$

(see Gurtin [15]).

Computing the mobility by formula (2.8), with $\mathbf{q}$ being the two phase stationary wave solution, gives for unit vectors $v$

$$
\mu_{i j}(v)=\tilde{\mu}_{i j} \frac{\beta_{0}+B_{i j}^{s m}(v)}{A_{i j}^{s m}(v)}
$$

and

$$
\hat{\mu}_{i j}(\theta)=\tilde{\mu}_{i j} \frac{\beta_{0}+\beta_{i j} \cos \left(L_{i j}\left(\theta-\gamma_{i j}\right)\right)}{1+\delta_{i j} \cos \left(M_{i j}\left(\theta-\alpha_{i j}\right)\right)} .
$$

We remark that the formulas (2.17) and (2.18) are only true, if the two phase stationary wave solution, i.e. the solution which connects the two minimas on a straight line, and the $N$-phase stationary wave solution are the same (see (2.7) and (2.8)). In [14], we observed with the help of numerical simulations that this is the case to a sufficient accuracy, when the $\sigma_{i j k}$ in $\hat{\Psi}_{o b}$ are sufficiently large.

- Crystalline anisotropies

For crystalline anisotropies the corresponding Wulff shape is a convex polygon (see Gurtin [15], Taylor [30]). A polygonial Wulff shape is possible for certain non-differentiable surface energies. We consider crystalline energies of the form

$$
\sigma(v):=\max \left\{v \cdot \eta^{k} ; k=1, \ldots, n\right\},
$$


where $\left\{\eta^{1}, \ldots, \eta^{n}\right\}$ are given. Then, the corresponding Wulff shape

$$
\mathcal{W}:=\left\{x \mid \quad x \cdot y \leqslant \sigma(y) \text { for all } y \in \mathbb{R}^{2}\right\}
$$

is the convex hull of $\left\{\eta^{1}, \ldots, \eta^{n}\right\}$ (see Appendix).

For the crystalline phase field, we therefore choose the gradient energy coefficients

$$
A_{i j}^{c r}(y):=\max \left\{(y /|y|) \cdot \eta_{i j}^{k} ; k=1, \ldots, n_{i j}\right\}
$$

and the mobility coefficients

$$
B_{i j}^{c r}(y):=\max \left\{(y /|y|) \cdot \zeta_{i j}^{k} ; k=1, \ldots, m_{i j}\right\},
$$

with $\eta_{i j}^{k}, \zeta_{i j}^{k} \in \mathbb{R}^{2}$ and $n_{i j}, m_{i j} \in \mathbb{N}$.

\section{Numerical simulations}

In this section, we present results from numerically solving the initial boundary value problem to (2.11) on a rectangular domain: $\Omega=(0, a) \times(0, b)=\left(0, N_{x} h\right) \times\left(0, N_{y} h\right)$ with a cell size $h$ and with $N_{x}$ and $N_{y}$ the number of cells in x- and in y-directions. To discretize the multi-phase field model we want to use the fact that the system has a gradient flow structure. Using forward differences

$$
\nabla_{+}^{h} \mathbf{u}=\frac{1}{h}(\mathbf{u}(x+h, y)-\mathbf{u}(x, y), \mathbf{u}(x, y+h)-\mathbf{u}(x, y))
$$

as approximation to $\nabla \mathbf{u}$ we discretize the Ginzburg-Landau energy functional as

$$
\mathcal{E}^{h}(\mathbf{u}):=\sum_{k, l=0}^{N_{x}-1, N_{y}-1}\left[\varepsilon f_{\text {aniso }}\left(\mathbf{u}(k h, l h), \nabla_{+}^{h} \mathbf{u}(k h, l h)\right)+\frac{1}{\varepsilon} \hat{\Psi}_{o b}(\mathbf{u}(k h, l h))+\Phi(\mathbf{u}(k h, l h))\right] h^{2}
$$

where $\mathcal{E}^{h}$ is defined for functions defined on the grid $G^{h}:=\left\{(k h, l h) \in \mathbb{R}^{2} \mid k=0,1, \ldots, N_{x} ; l=\right.$ $\left.0,1, \ldots, N_{y}\right\}$ with values in $\Sigma$.

Now a solution $\mathbf{u}: G^{h} \times(0, T) \rightarrow \mathcal{G}$ of the discrete $L^{2}$-gradient flow by definition has to fulfill

$$
\varepsilon\left(\beta\left(\mathbf{u}, \nabla^{h} \mathbf{u}\right) \mathbf{u}_{t}, \mathbf{v}-\mathbf{u}\right)(t)+\left(D \mathcal{E}^{h}(\mathbf{u}), \mathbf{v}-\mathbf{u}\right)(t) \geqslant 0,
$$

for all $\mathbf{v}: G^{h} \times(0, T) \rightarrow \mathcal{G}$ and $t \in(0, T)$. Here we denote by (., .) the eucledian scalar product on $G^{h}$ and $D \mathcal{E}^{h}$ is the gradient of $\mathcal{E}^{h}$. To discretize $\nabla \mathbf{u}$ in the $\beta$ term we used $\nabla^{h} \mathbf{u}(x, y):=$ ( $\left.\partial_{x}^{h} u(x, y), \partial_{y}^{h} u(x, y)\right)$ where $\partial_{x}^{h} u(x, y)\left(\partial_{y}^{h} u(x, y)\right.$ respectively) is the forward difference quotient with respect to the $x$-variable ( $y$-variable respectively) whenever a corresponding neighbour to compute the quotient exists and the backward difference quotient at all other points.

For the sake of simplicity, we use an explicit finite difference algorithm to solve (3.1) numerically with the time step given by $\Delta t$. If $\mathbf{u}^{m-1}$ is the solution computed at the time $(m-1) \Delta t$ we determine the solution $\mathbf{u}^{m}$ at time $m \Delta t$ as the solution of

$$
\varepsilon\left(\beta\left(\mathbf{u}^{m-1}, \nabla^{h} \mathbf{u}^{m-1}\right) \frac{\mathbf{u}^{m}-\mathbf{u}^{m-1}}{\Delta t}, \mathbf{v}-\mathbf{u}^{m}\right)+\left(D \mathcal{E}^{h}\left(\mathbf{u}^{m-1}\right), \mathbf{v}-\mathbf{u}^{m}\right) \geqslant 0
$$


which has to hold for all $\mathbf{v}: G^{h} \rightarrow \mathcal{G}$. This inequality can be solved by first setting

$$
\overline{\mathbf{u}}^{m}:=\mathbf{u}^{m-1}-\Delta t\left(\varepsilon \beta\left(\mathbf{u}^{m-1}, \nabla^{h} \mathbf{u}^{m-1}\right)\right)^{-1} T D \mathcal{E}^{h}\left(\mathbf{u}^{m-1}\right)
$$

and then defining on each grid point $(k h, l h)$ the value $\mathbf{u}^{m}(k h, l h)$ to be the projection of $\overline{\mathbf{u}}^{m}$ onto the Gibbs simplex $\mathcal{G}$ (see for example [8] and [20] for the scalar case).

To determine $D \mathcal{E}^{h}$ we need to differentiate $f$. With $N_{i j}:=u_{i} \nabla u_{j}-u_{j} \nabla u_{i}$ and $T_{i j}:=N_{i j}^{\perp}$, where $N_{i j}^{\perp}$ is the vector $N_{i j}$ rotated by $90^{\circ}$ degree, we get

$$
f_{, u_{i}}=2 \sum_{j \neq i} \frac{\tilde{\sigma}_{i j}}{\tilde{\mu}_{i j}} A_{i j}\left(N_{i j}\right) \nabla u_{j} \cdot\left[A_{i j}\left(N_{i j}\right) N_{i j}+\left(A_{i j}\right)_{\theta}\left(N_{i j}\right) T_{i j}\right]
$$

and

$$
f_{, X_{i}}=-2 \sum_{j \neq i} \frac{\tilde{\sigma}_{i j}}{\tilde{\mu}_{i j}} A_{i j}\left(N_{i j}\right) u_{j}\left[A_{i j}\left(N_{i j}\right) N_{i j}+\left(A_{i j}\right)_{, \theta}\left(N_{i j}\right) T_{i j}\right] .
$$

It can be seen that the term $T D \mathcal{E}^{h}\left(\mathbf{u}^{m-1}\right)$ appearing in (3.3) is equivalent to a finite difference approximation of the right hand side in (2.2) where the gradients are discretized by forward differences and the divergence is discretized by backward differences. We point out that discretizing first the Ginzburg-Landau energy and then taking the gradient of the energy gives an easy way to obtain a consistent discretization of the boundary condition in a finite difference framework. Of course, also a finite element approximation could be used.

As values for the parameters, if not otherwise stated, we choose $a=b=1, \epsilon=1 / 10, h=$ $\epsilon / 10, \Delta t=h^{2} / 10, \tilde{\mu}_{i j}=1, \tilde{\sigma}_{i j}=1, \sigma_{i j k}=5$ and $m_{i}=0$.

The remainder of this section is divided into two parts. In the first subsection, we consider the evolution of phase boundaries and junctions under smooth anisotropies and in the second subsection, we present simulations with crystalline anisotropies.

The numerical results are presented in the following way: In several figures, e.g. in Fig. 3, we show the values of one phase-field variable $u_{i}$ in the whole domain by using different grey shades. In the interfacial region a smooth transition from $u_{i}=1$ to $u_{i}=0$ can be observed. For representations such as Fig. 8, we divide the Gibbs simplex into $N$ parts. Phase $i$ or grain $i$ is represented by all $\mathbf{u}$ with $u_{i}$ bigger than the other components. A special colour is used to display the different phases or grains. Then, the domain $\Omega$ is divided into subregions, displayed by different colours, according to the values of $\mathbf{u}$ in the numerical cells. In other Figures, we display interfacial curves (see Fig. 1). Here, we define interfacial cells to be cells with a neighbouring cells of different colour. Applying this procedure, the position of the interfaces is determined and drawn in images such as Fig. 1.

\subsection{Smooth anisotropy}

To study the effect of smooth anisotropy, we use the formulation (2.15) and (2.16) in which the surface free energy and the kinetic coefficient vary smoothly with the orientation of the interfacial contours. In the following, we describe the influence of varying different parameters that control the characteristics of anisotropy. If not otherwise stated, we set $\beta_{0}=1$ and $\beta_{i j}=0$ for the kinetic coefficient. The simulations displayed in Figs 1 to 7 , are for systems with two phases initially, computed in a setting with three order parameters. One observes that the third order parameter remains zero and hence, only two phases are present during the evolution. 
- Interfaces with smooth anisotropy in the interior of the domain.

In Fig. 1, we present the results of our calculations for a family of smooth anisotropies.

Next, we demonstrate that the computational square mesh has no influence on the calculated orientation of the grains. For this, in the case of four-fold anisotropy, we choose different values of the relative angle $\alpha_{i j}$. The interface evolution is shown in Fig. 2. Slight undulations are a result of a plotting effect.

In the experiments of Fig. 3, we study the influence of the strength parameter $\delta_{i j}$ on the sharpness of the crystal vertices for $M_{i j}=4, \alpha_{i j}=30^{\circ}$, while keeping all other parameters in the system constant. We remark that, for

$$
\left|\delta_{i j}\right|>\frac{1}{M_{i j}^{2}-1},
$$

the anisotropy (2.15) is unstable and that the Wulff shape which belongs to $\sigma_{i j}$ has vertices (see [15]). Corners are observed in (b) and (c) of Fig. 3, where $\delta_{i j}$ is larger than the critical value.

a
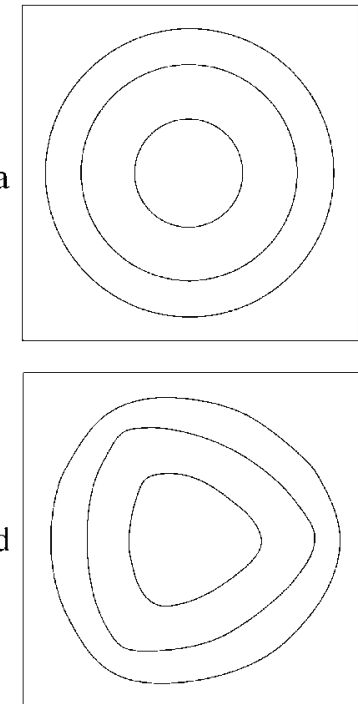

b
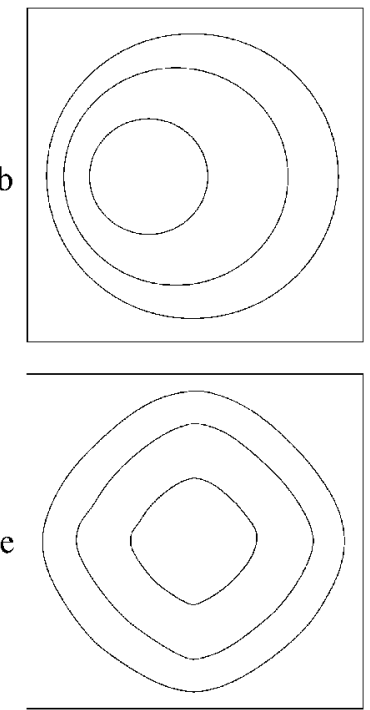

c
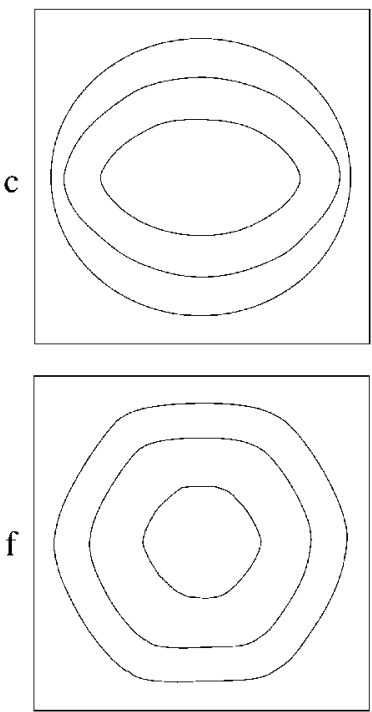

FIG. 1. Dissolution of a crystal at time steps $t=0.01,0.04,0.07$ (a) of an isotropic crystal, (b)-(f) of a crystal with surface energy anisotropy with the parameters $\alpha_{i j}=0^{\circ}, M_{i j}=1,2,3,4,6$ and corresponding $\delta_{i j}=0.5,0.3,0.12,0.065,0.025$.

The snapshots of Fig. 4 illustrate that the interfacial thickness depends on the orientation of the interface. This feature is always observed, if surface energy anisotropy is considered, since the thickness of the interface is directly proportional to $A_{i j}(v)$ (see [13]). In the case $M_{i j}=4$ of Fig. 3 , a change in interfacial thickness along the interface also occurs, but on a much smaller lengthscale, so that the effect cannot be detected as easily as in the case $M_{i j}=1$. 

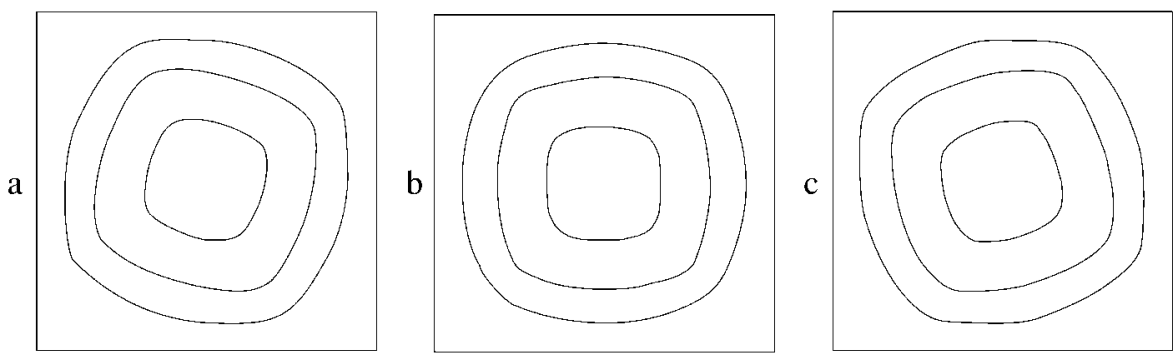

FIG. 2. Crystals of different orientation with respect to the numerical grid, (a) $\alpha_{i j}=30^{\circ}$, (b) $\alpha_{i j}=45^{\circ}$, and (c) $\alpha_{i j}=60^{\circ}$ at time steps $t=0.01,0.04,0.07$.
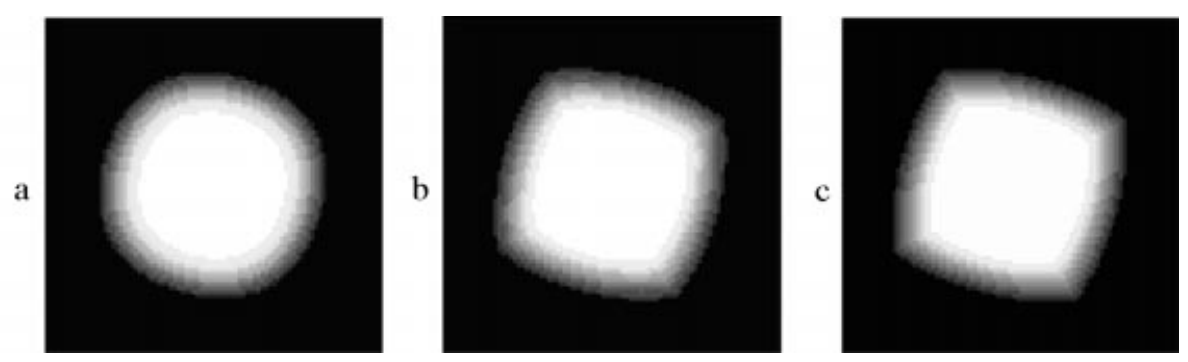

FIG. 3. The sharpness of the crystal vertices increases with the anisotropy strength parameter (a) $\delta_{i j}=0.02$, (b) $\delta_{i j}=0.08$ and (c) $\delta_{i j}=0.15$. The three plots depict the same time step.

In contrast to the previous observations, we observe that, in numerical simulations with $L_{i j}=1$ and $\beta_{i j}=0.5$, the interfacial thickness remains the same all along the crystal surface if anisotropy is purely introduced within the time relaxation parameter while keeping the surface energy isotropic.

Anisotropy may be introduced via the surface energy $\sigma$ or the kinetic coefficient $\beta$. The table in Fig. 5 summarizes the impact of either choice. If the anisotropy is introduced in $\sigma$ the profiles for growing and shrinking roughly have the same shape, whereas if the anisotropy is introduced in $\beta$, the profiles for growing and shrinking are different. Our results are in accordance with a theoretical result by Soner [24]. In his work, he showed that the asymptotic shape for a growing interface is given by the Wulff shape of $1 / \mu_{i j}$.

- Effects of a smooth interfacial anisotropy at the domain boundaries.

Now, we direct our attention to the situation in which interfaces intersect the domain boundary. In the simulation displayed in Fig. 6, the interface was initially set as a sharp cosine profile with $M_{i j}=2, \alpha_{i j}=60^{\circ}, \delta_{i j}=0.3$. Once the computation is started, the interface becomes diffuse and the evolution shows that the angle between the interface and the domain boundary very rapidly changes (see snapshots with higher magnification of the region close to the boundary). The deviation from the $90^{\circ}$ angle condition of the standard isotropic case is due to the Herring torque term 

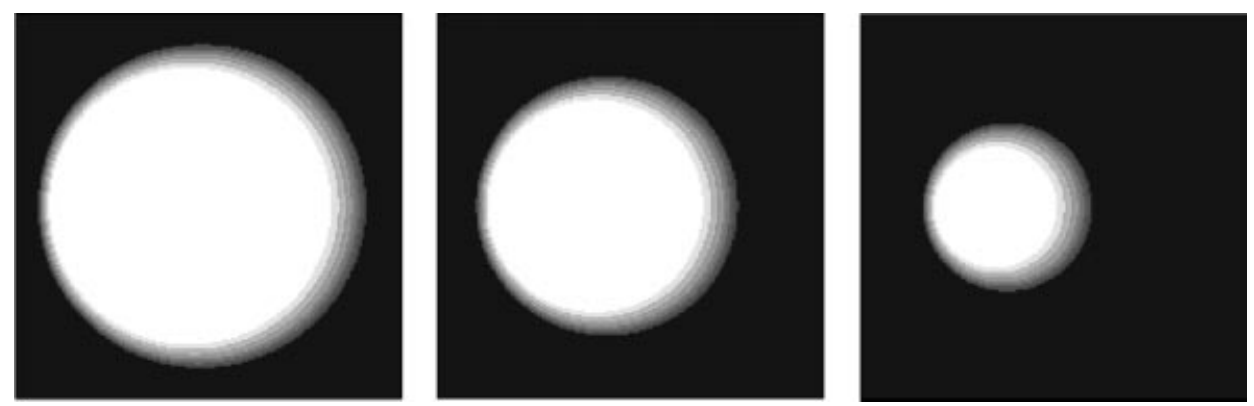

FIG. 4. Time evolution of a crystal with anisotropic surface energy $\left(M_{i j}=1, \delta_{i j}=0.5\right)$ with locally varying interfacial thickness at time $t=0.09,0.18,0.27$.

$\hat{\sigma}_{i j}^{\prime}(\theta) \tau$. This term enters (2.6) in the anisotropic case. Therefore, an angle related to surface energy anisotropy adjusts at the domain boundary.

From (2.6), we obtain the following equation for the angle $\varphi$ that an interface forms with the right hand boundary

$$
\left(1+\delta_{i j} \cos \left(M_{i j}\left(\varphi-\alpha_{i j}\right)\right)\right) \cos \varphi+\delta_{i j} M_{i j} \sin \left(M_{i j}\left(\varphi-\alpha_{i j}\right)\right) \sin \varphi=0 .
$$

The angle $\varphi$ is marked in the last plot of Fig. 6 and the solution of (3.1) is $\varphi \approx 121.1353$. We solved the discretized phase field equations for different mesh sizes $h$ and determined the angle $\varphi(h)$ of the numerical solution at time $t=0.8$. The values obtained are: $\varphi\left(\frac{1}{100}\right)=120.54, \varphi\left(\frac{1}{200}\right)=121.0475$ and $\varphi\left(\frac{1}{300}\right)=121.1039$.

The influence of anisotropy at the domain boundary is further illustrated in another numerical experiment with $M_{i j}=2, \alpha_{i j}=120^{\circ}, \delta_{i j}=0.3$. The simulation is carried out with an initially planar front orientated in horizontal direction, so that the interface meets the domain boundary at $90^{\circ}$. The anisotropic nature of the surface energies causes forces that act on the interface and rotate the interface towards a direction with lower energy (see Fig. 7). In a contrasting experiment, we observed that an interface with isotropic energy, initially meeting the domain boundary with an angle that differs from $90^{\circ}$, evolves and finally attains a perpendicular intersection with the domain boundary. In another numerical computation, we found that an anisotropic time relaxation parameter has no influence on the angle at the domain boundary.

- Simulation of trijunctions with smooth anisotropic surface energies.

We performed a series of simulations of anisotropic trijunction evolutions which support our asymptotic analysis (see [13]). In Fig. 8 we display two examples. Initially, we set three phases in a configuration, so that their interfaces meet at the trijunction with $120^{\circ}$ angles. The initial setup is illustrated with black lines in the first plots of Fig. 8(a) and (b). Young's law is violated at the triplejunction due to additional shear forces which result from the anisotropy within the surface energies. These forces, known as Herring torque terms, act in the normal directions to the interfaces and cause the trijunction to vary rapidly in order to adjust with angles that correspond to an anisotropic force balance. 


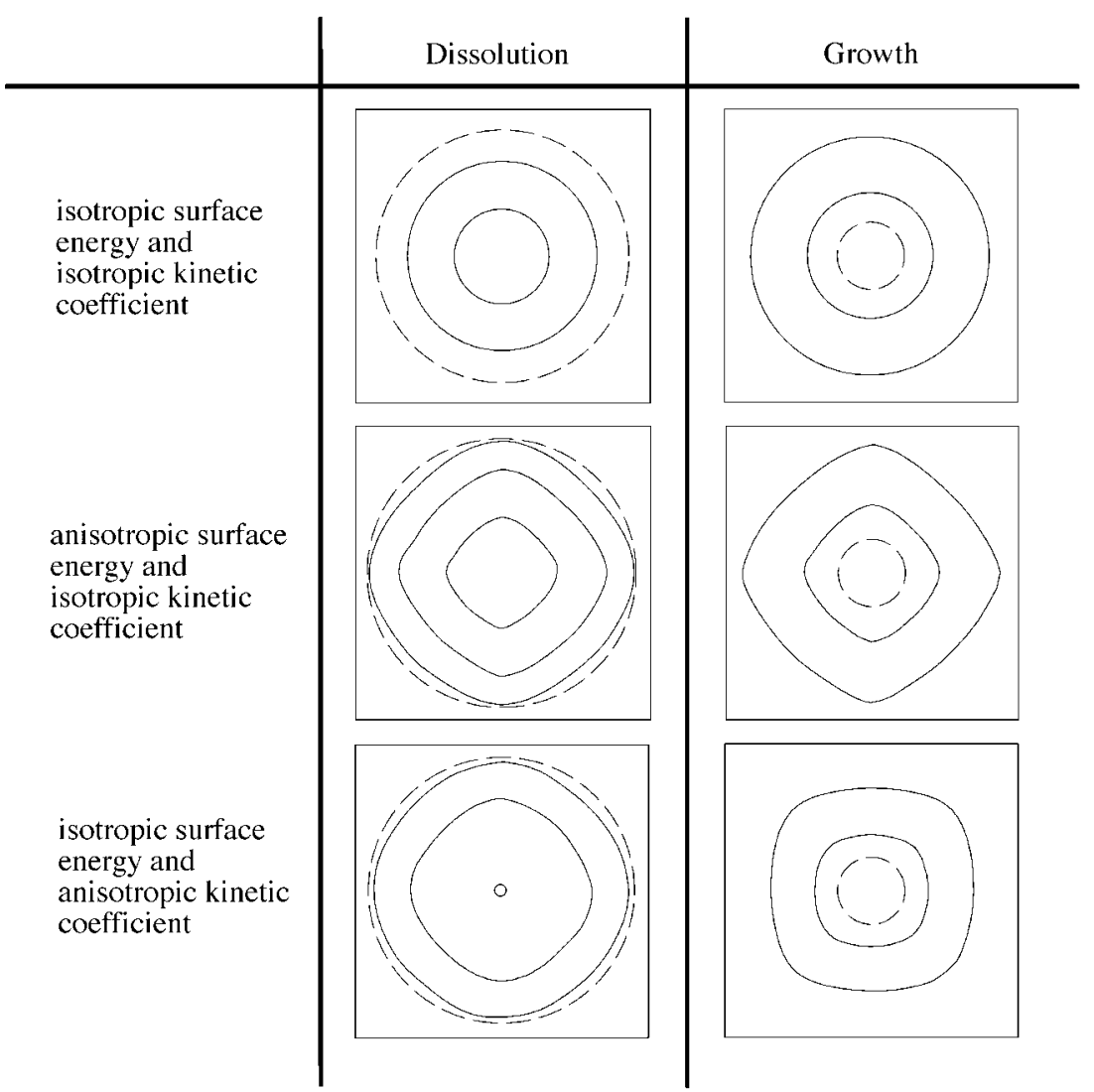

FIG. 5. For anisotropic $\sigma_{i j}: M_{i j}=4, \delta_{i j}=0.065, \alpha_{i j}=0^{\circ}$, for anisotropic $\beta: L_{i j}=4, \beta_{i j}=0.5, \gamma_{i j}=0^{\circ}$, for growing: $m_{j}=12, t=0.00,0.02,0.04$, for shrinking: $t=0.01,0.04,0.07$ (dashed lines: initial profile).

Using the set of parameters $M_{i j}=2, \tilde{\sigma}_{12}=1.0, \tilde{\sigma}_{13}=0.9, \tilde{\sigma}_{23}=1.6, \delta_{12}=0.3, \delta_{13}=$ $0.3, \delta_{23}=0.0$ and $\alpha_{i j}=0^{\circ}$, we established an example in which a trijunction breaks down (see Fig. 9). The surface energies were chosen such that there is the possibility of finding angles $\theta_{1}, \theta_{2}$ and $\theta_{3}$ such that Young's law (2.5) can be fulfilled. We started with $T$-shaped initial data which were rotated by $90^{\circ}$. This is a configuration which does not fulfill Young's law and we wanted to check numerically whether the triple junction readjusts itself to attain angles that fulfill Young's law, i.e. to fulfill the local equilibrium condition. Instead of locally changing the angles towards a configuration which fulfills Young's law, which for our choice of surface energies would be possible, the evolution builds up a channel of phase 1 between the phases 2 and 3 . This can be interpreted as a wetting phenomenon (see Cahn [5] and Kikuchi \& Cahn [18]). An isotropic experiment with surface energies which violate the stability condition

$$
\sigma_{i j} \leqslant \sigma_{j k}+\sigma_{k i}
$$



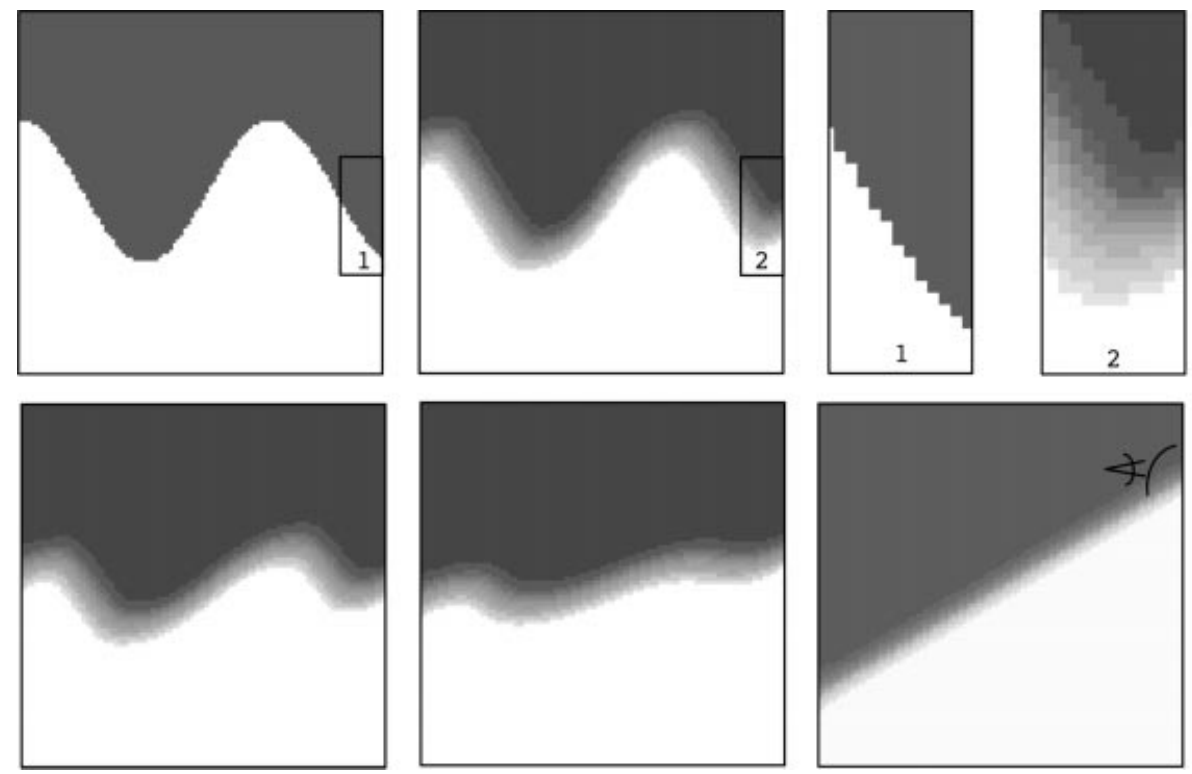

FIG. 6. Evolution of an initially curved interface with smooth anisotropy at time steps $t=$ $0.000,0.005,0.015,0.030,0.80$ and a higher magnification of the interfacial contour lines at the right hand domain boundary.
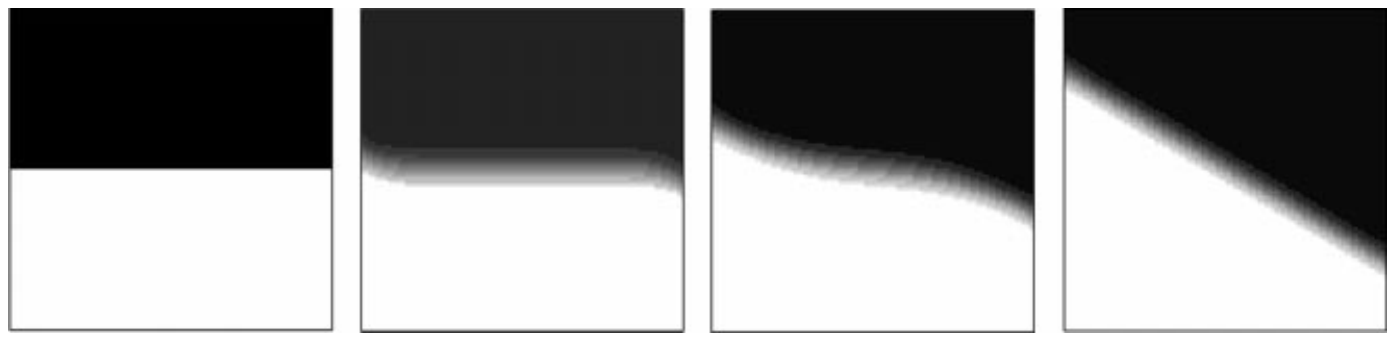

FIG. 7. A diffuse interface with anisotropic surface energy shown at time steps $t=0.000,0.005,0.050,0.450$.

also would give rise to wetting. In our case anisotropic surface energies were chosen such that there are directions in which the stability condition is fulfilled and directions in which it is violated. This means in principle the system could vary locations locally in order to fulfill Young's law. But our simulations demonstrate that also in this case wetting occurs.

\subsection{Crystalline anisotropies}

We expect that the phase field model with the crystalline anisotropy, as introduced in Section 2, approximates motion by crystalline curvature. Motion by crystalline curvature was introduced independently by Taylor [29] and Angenent \& Gurtin [1]. In the following, we want to show that 
a
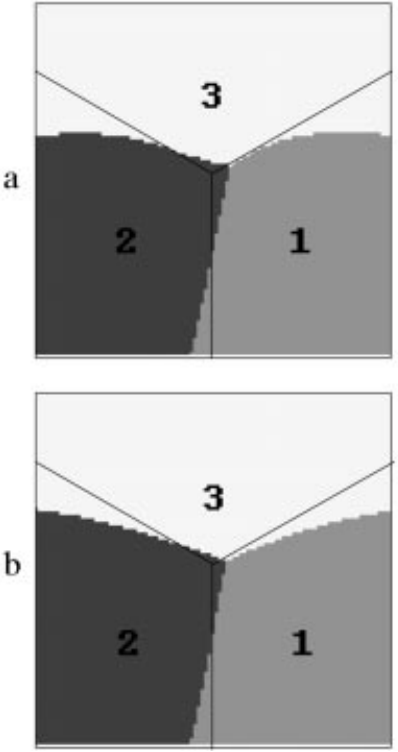
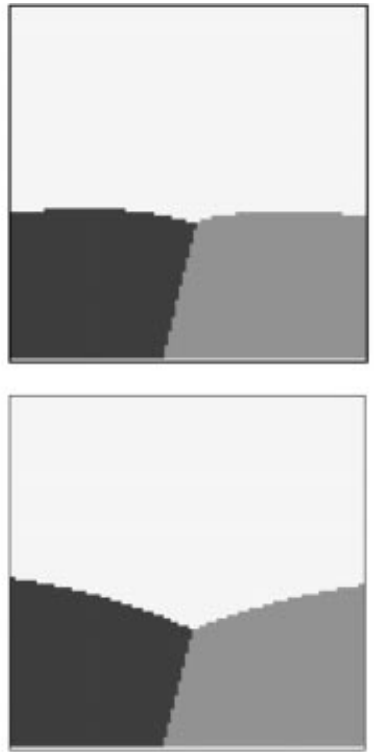
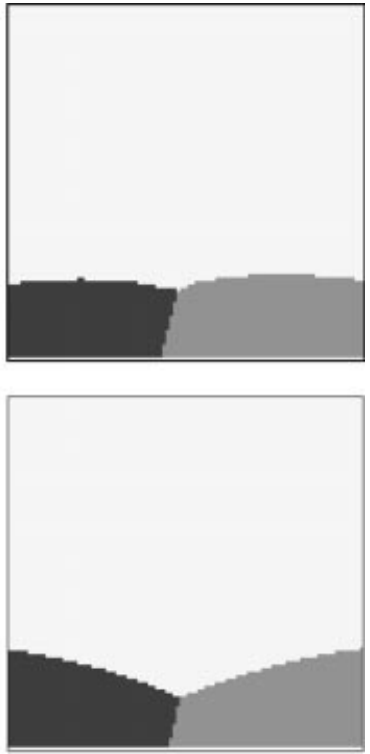

FIG. 8. Motion of trijunctions at time steps $t=0.05,0.25,0.45$ and with parameters $\delta_{i j}=0.065, M_{i j}=4$. The relative angles are (a) $\alpha_{12}=15^{\circ}, \alpha_{13}=60^{\circ}, \alpha_{23}=30^{\circ}$ and (b) $\alpha_{12}=15^{\circ}, \alpha_{13}=30^{\circ}, \alpha_{23}=60^{\circ}$.
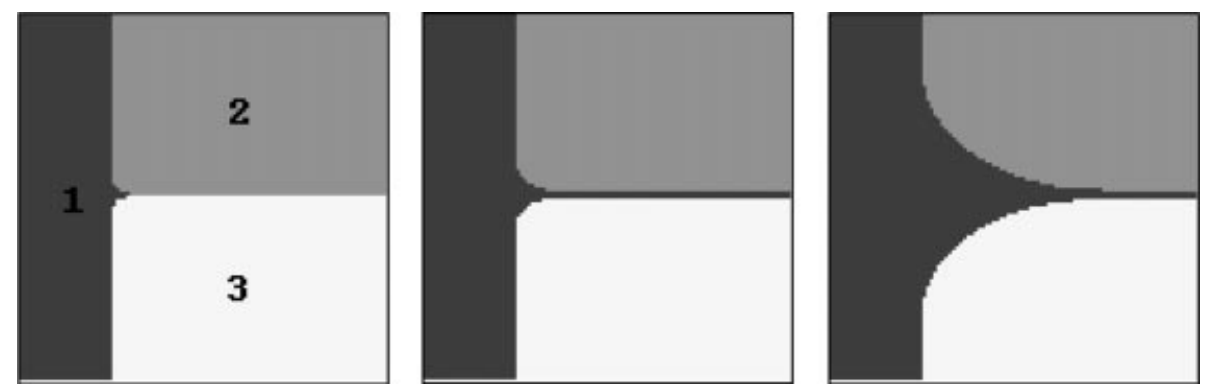

FIG. 9. Simulation of an unstable trijunction at time steps $t=0.0005,0.0200,0.0300$.

it is possible to use the phase field model to approximate motion by crystalline curvature in the multi-phase case. For a precise definition of the crystalline flow, we refer to [15] and [30] and, for results on the two phase crystalline Allen-Cahn equation, we refer to [2].

In this paragraph, we state results from numerical simulations with crystalline anisotropy (see (2.20) and (2.21)). In this case, $A_{i j}$ is non-differentiable. Hence, the Allen-Cahn system as it is formulated in Section 2 is not well defined. To still numerically treat the crystalline anisotropy, we proceed as follows. In the algorithm, we need to assign values to $f_{, u_{i}}$ and $f_{, X_{i}}$ (see (3.4) and (3.5)), which means we have to specify a value for $\left(A_{i j}\right)_{, \theta}$ also at points where it is non-differentiable. Whenever $\left(A_{i j}\right)_{, \theta}$ jumps, we choose a value which lies in the jump interval. As we shall see in the 
following, this gives reasonable numerical results, but a rigorous justification for this procedure is still missing.

To obtain a crystalline energy coefficient $A_{i j}^{c r}$ with $M_{i j}$-fold symmetry and relative angle $\alpha_{i j}$ we set in (2.20) $n_{i j}=M_{i j}$ and

$$
\eta_{i j}^{k}:=\left(\cos \left(\frac{k}{M_{i j}} 2 \pi-\alpha_{i j}\right), \sin \left(\frac{k}{M_{i j}} 2 \pi-\alpha_{i j}\right)\right)
$$

for $k=1, \ldots, M_{i j}$. With this choice, one obtains a Wulff shape which is a regular polygon. In all the simulations we present in the crystalline case, we choose $\beta$ to be one.

To better compare the results, we examine similar situations as described in the previous subsection for smooth surface energy anisotropies.

- Interfaces with crystalline anisotropy in the interior of the domain.

In Fig. 10, we begin with single crystals located in the interior of the domain. The position of the initial interface is marked with dashed lines. During the growth into the melt, the shape of the crystals shows facets and sharp vertices due to the crystalline anisotropy. In Fig. 10b we choose the Wulff shape as initial data and we observe that it evolves without change in shape. This solution is known as Taylor's solution (see Taylor [29] and Gurtin [15]).
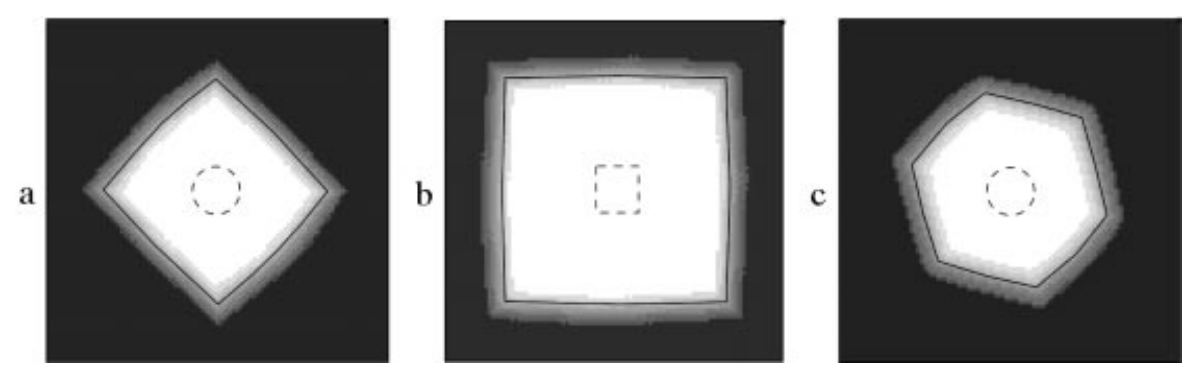

FIG. 10. Faceted crystal growth at time steps $t=0.00,0.03$ and with parameters (a) $M_{i j}=4, \alpha_{i j}=0^{\circ}, m_{j}=$ 12, (b) $M_{i j}=4, \alpha_{i j}=45^{\circ}, m_{j}=15$ and (c) $M_{i j}=6, \alpha_{i j}=45^{\circ}, m_{j}=12$.

- Effects of a crystalline interfacial anisotropy at the domain boundaries.

In Fig. 11, we investigate the behaviour of an interface with crystalline surface energy which intersects the domain boundary. We compare the observations with the results of Fig. 6, for smooth anisotropy. The system tends to form strong facets and only directions which appear in the Wulff shape are present after some time. It is interesting to study what the boundary conditions look like in the crystalline case. To our knowledge, no derivation of boundary conditions in the crystalline case have been made so far. If, locally at the boundary, interfacial energy is going to be minimized, the boundary condition looks as follows (see [12] for more details). There is a vector $\xi$ in the subdifferential $D \sigma(v)$ such that $\xi \cdot v_{\partial \Omega}=0$. In the case of a non-differentiable surface energy $\sigma$ 
the subdifferential $D \sigma(v)$ is a whole set and therefore, for the directions of the Wulff shape, there is a choice of vectors $\xi$. The vector $\xi^{\perp}$ is the capillary force in the sense of Gurtin [15] and the above condition says that there exists a shear force (see [15]), such that the total force is normal to the external boundary.
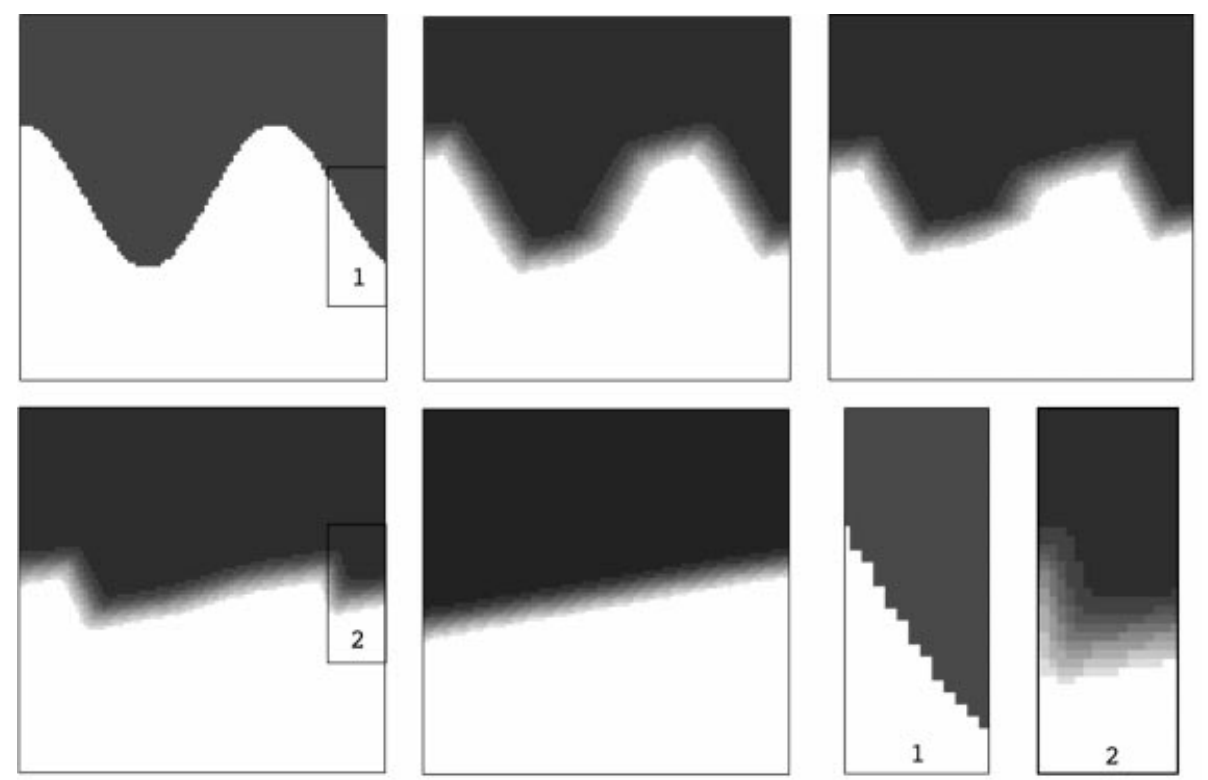

FIG. 11. Evolution of an initially curved interface with crystalline anisotropy $M_{i j}=4, \alpha_{i j}=60^{\circ}$ shown at time steps $t=0.000,0.005,0.015,0.030,0.450$ and a snapshot of the interfacial contour lines at the right hand domain boundary.

In Fig. 12, we started with a horizontally aligned planar interface and with $M_{i j}=4, \alpha_{i j}=10^{\circ}$. The local point of contact with the domain boundary begins to move in order to adjust to an angle that is suitable with respect to the system parameters. Sharp steps are formed within the interface. Under the constraint to keep an angle at the domain boundary which fulfills the force balance, the system minimizes the crystalline surface energy. The behaviour may be compared with Fig. 7. One clearly observes that the facet in the middle, which is neither convex nor concave with respect to the neighbouring facets has zero velocity as it should be according to motion by crystalline curvature. Only facets which are convex or concave with respect to their neighbours move and their velocity is inverse proportional to their length. This can be observed in the evolution of the interface illustrated in Fig. 12. For facets which intersect the external boundary, this usual law has to be modified in a suitable way and we address this issue in [12].

- Simulation of trijunctions with crystalline surface energies

For the numerical experiments of crystalline trijunctions (Figs 13 and 14), we used a T-shaped initial configuration indicated by black lines in Fig. 13. As a result of strong anisotropy, we obtain 

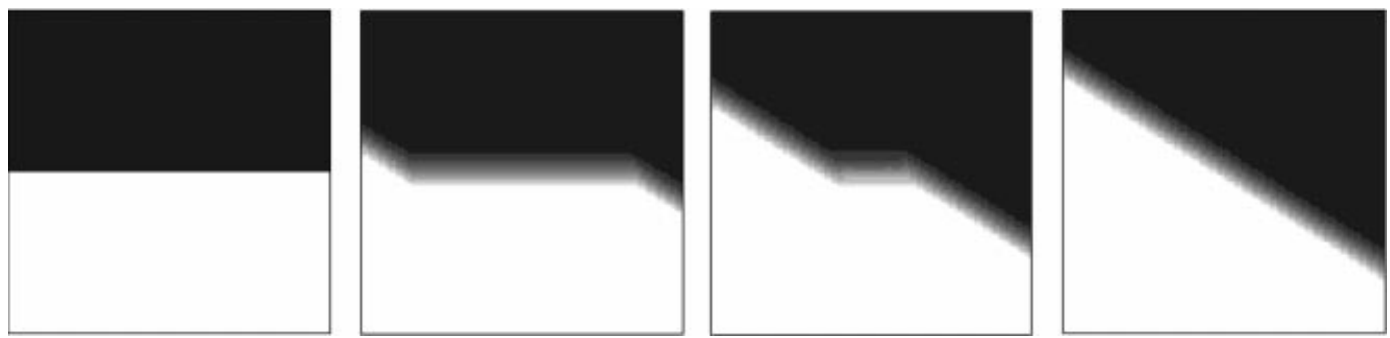

FIG. 12. Crystalline interface shown at time steps $t=0.00,0.05,0.30,0.55$.

sharp vertices along the interfaces and angle conditions that differ from $120^{\circ}$. The equilibrium condition at a trijunction can be formulated as follows. Let $v_{12}, v_{23}$ and $\nu_{31}$ be the three normals at the trijunction. If there exist vectors $\xi_{i j} \in D \sigma_{i j}\left(v_{i j}\right)$ such that

$$
\xi_{12}+\xi_{23}+\xi_{31}=0,
$$

then we say that the triple-junction is in local equilibrium. This condition appears if the surface energy local to a trijunction is minimized (see [12]). In this context, the equilibrium condition at the triple-junction can be interpreted as follows. There exist shear forces on the three interfaces such that the sum of the total forces at the triple junction is zero (see [12]). We remark that all triple-junctions that appear in the simulations illustrated in Figs 13-15 fulfill this equilibrium condition. The triple junction shown in Fig. 13 has a $90^{\circ}$ and two $135^{\circ}$ angles which fulfill the local equilibrium condition (3.6). We performed numerical simulations on different meshes and found that for all meshes the angles where attained locally at the triple junction.
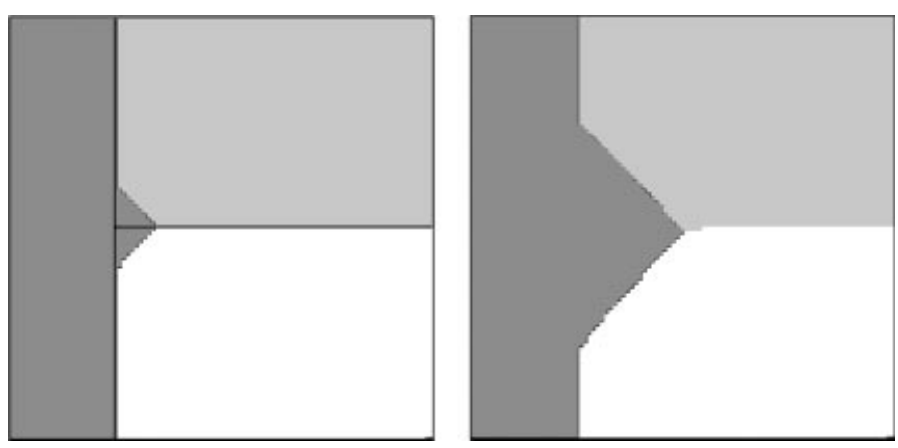

FIG. 13. Simulation of a crystalline trijunction with $M_{i j}=4, \alpha_{i j}=0^{\circ}, t=0.008,0.064$.

- Symmetries of grain boundary trijunctions

To demonstrate the applicability of the crystalline version of the phase field method, we model the evolution of grain boundaries and trijunctions in tricrystalline aluminum films. In certain 

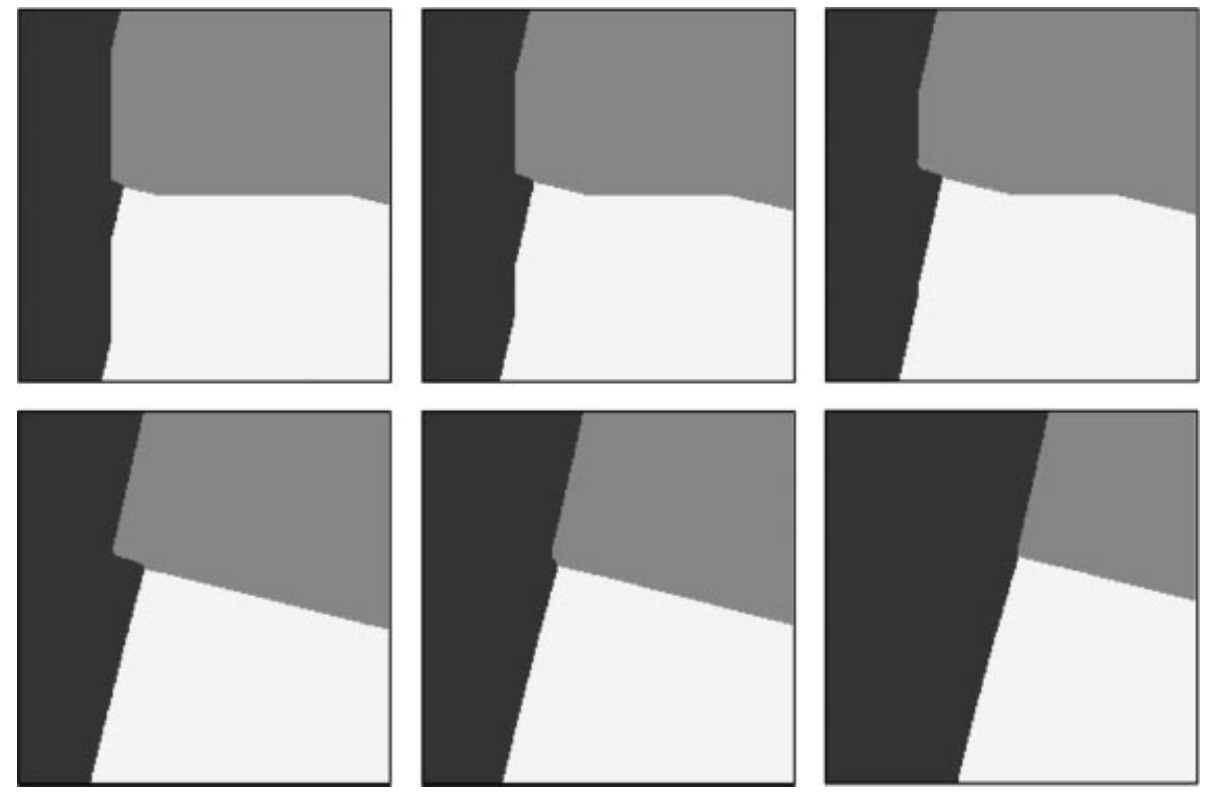

FIG. 14. Simulation of a crystalline trijunction with $M_{i j}=4, \alpha_{i j}=30^{\circ}, h=0.005, t=0.004$, $0.010,0.016,0.048,0.144,0.432$.

experiments of heteroepitaxial thin aluminum film growth on a silicon substrate, two families of triple-junctions with different symmetries are observed (see [31,32]). Using the principle of symmetry dictated extrema Cahn \& Kalonji [6] classified the different triple-junctions in these films. It was shown that triple junctions fall into two families, one being highly symmetric and one being less symmetric. In addition, they obtained that neighbouring triple-junctions must belong to different symmetry classes. John Cahn asked the question whether it is possible to model these systems by a phase-field model. In particular, it is important to recover the angle conditions and, hence, the symmetries at trijunctions correctly.

We study such a situation numerically by using the crystalline formulation of anisotropy and phase-field simulations. We use arguments of grain boundary symmetry to specify the expression for crystalline surface energy anisotropy. The derivation is explained in detail in [12]. There, we discuss that an eight-fold anisotropy is a suitable choice of the crystalline surface energy anisotropy to model the observed tricrystalline growth structures. The motivation for considering symmetry aspects is based on geometrical and topological properties examined in [6] by Cahn \& Kalonji.

We work with an asymmetric initial phase configuration shown in Fig. 15(a), with a crystalline eighth-fold anisotropy $\left(M_{i j}=8\right)$, with $h=0.0033$ and with relative angles $\alpha_{12}=$ $7.5^{\circ}, \alpha_{13}=22.5^{\circ}$ and $\alpha_{23}=37.5^{\circ}$. With this set of parameters, the simulation recovers the observations made in experiments. A symmetric $\left(120^{\circ}, 120^{\circ}, 120^{\circ}\right)$ triple-junction and a less symmetric $\left(150^{\circ}, 105^{\circ}, 105^{\circ}\right)$ adjacent triple-junction remain stable. Here, Fig. 15 corresponds to the experimental picture of figure 2 in [31] (see also figure 1 in [6]). Figure 15 illustrates one of a set of numerical experiments which show that it is possible to model crystalline grain boundaries with a multi-phase-field model and therefore answers Cahn's question. 

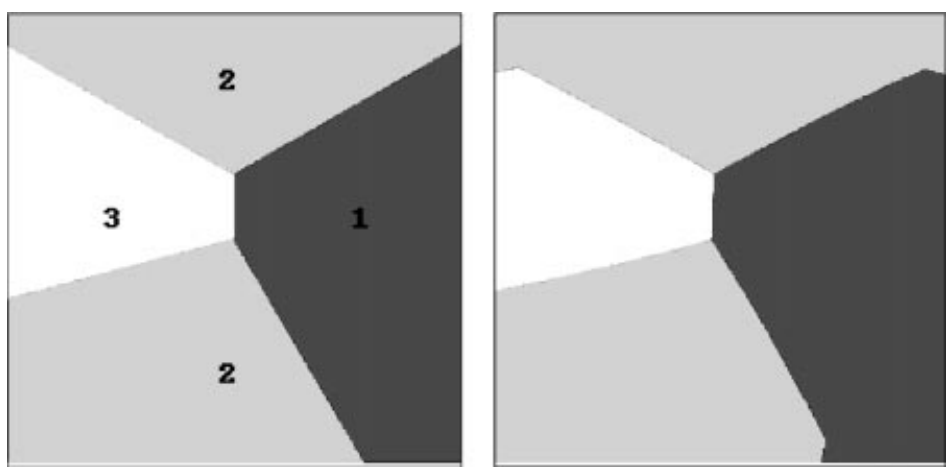

FIG. 15. Symmetries of grain boundary trijunctions. The angles at the two trijunctions are $120^{\circ}, 120^{\circ}, 120^{\circ}$ and $150^{\circ}, 105^{\circ}, 105^{\circ}$, respectively.

- Crystalline grain growth

In a final simulation, we apply the multi-phase-field model to grain growth phenomena. In this context, the components of $\mathbf{u}$ represent grains of the same phase, but of different crystallographic orientations. The simulation depicted in Fig. 16 is performed with four order parameters, with $h=0.0033, N_{x}=N_{y}=300, \epsilon=0.02, \Delta t=0.0000015$ and $M_{i j}=6$. The evolution of the grain boundaries support the fact that facets form in certain preferred directions and that anisotropy changes the equilibrium angle condition of $120^{\circ}$.

\section{Conclusion}

We introduced a general multi-phase-field concept and stated its sharp interface limit. In this limit, the anisotropic expression of the Gibbs-Thomson law and of the Young's force balance can be derived. We gave expressions for the free energies to incorporate both, smooth and crystalline anisotropy.

In the main part of this article we described numerical simulations using the above model. After briefly outlining the numerical schemes and discretizations, we have presented the following list of numerical results:

\section{Smooth anisotropy:}

- Interfaces in the interior of the numerical domain:

We investigated different features of the growth- and dissolution-behaviour in two-phase systems. In particular, we studied the influence of different anisotropy parameters such as $M_{i j}, \alpha_{i j}$ (relative angle) and $\delta_{i j}$ (anisotropy strength) and found a good agreement with theoretical predictions.

- Interfaces at the domain boundary:

In these numerical computations, we demonstrated the influence of shear forces due to 


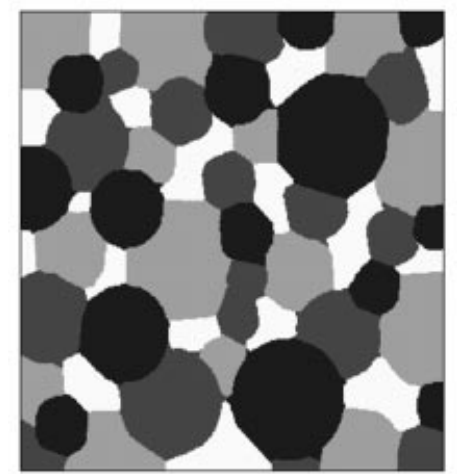

$\mathrm{t}=0.00045$

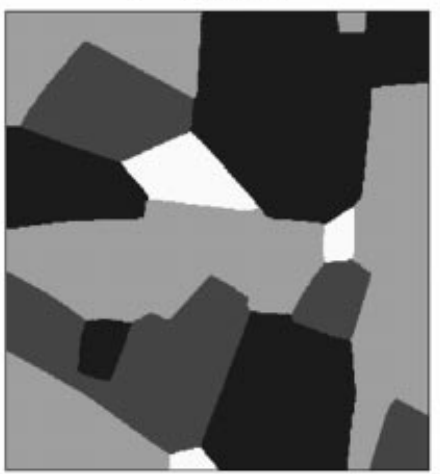

$\mathrm{t}=0.02025$

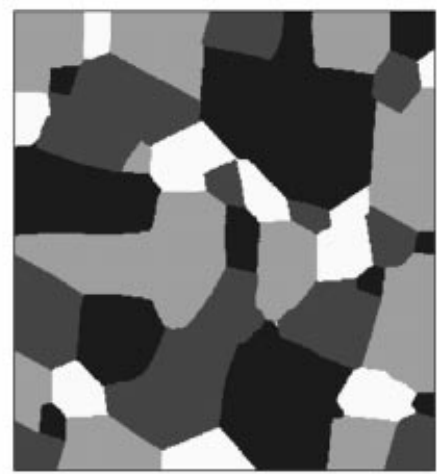

$t=0.00675$

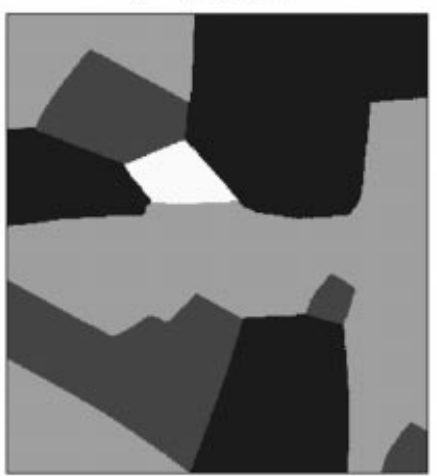

$\mathrm{t}=0.02700$

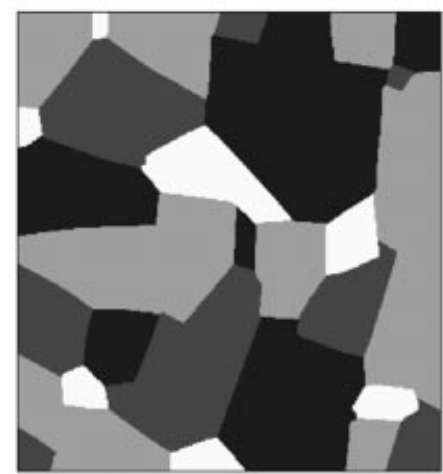

$\mathrm{t}=0.01350$

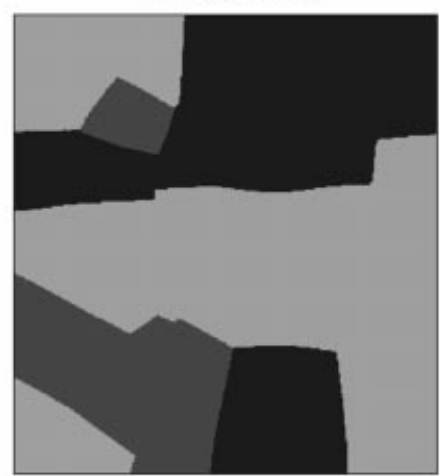

$\mathrm{t}=0.04725$

FIG. 16. Growth of grains with a six-fold anisotropy and with relatives angles: $\alpha_{12}=30^{\circ}, \alpha_{13}=45^{\circ}, \alpha_{14}=$ $90^{\circ}, \alpha_{23}=15^{\circ}, \alpha_{24}=60^{\circ}, \alpha_{34}=45^{\circ}$.

surface energy anisotropy and compared the numerically measured angle between the interface and the domain boundary with the appropriate analytical formula. We found a high agreement.

- Triple-junctions:

We performed simulations of anisotropic triple-junctions and observed angle conditions differing from the equilibrium $120^{\circ}$ condition. Furthermore, we gave an example for a wetting phenomenon.

\section{Crystalline anisotropy:}

- Interfaces in the interior and at the boundary of the numerical domain:

The numerical results illustrated the formation of sharp steps and strong facets while the interfaces evolve in time. With the tendency of the system to minimize crystalline surface energy, facets appear only in directions associated with the Wulff-shape directions. 
- Triple-junctions:

The simulations of crystalline triple-junctions showed the influence of shear forces at the interfaces. The multi-phase-field was applied to examine via numerical computations the symmetry properties of neighbouring triple-junctions that are observed in heteroepitaxial growth of thin film aluminum films. With the numerical result that two adjacent triplejunctions belong to different symmetry classes, our computations support the statements by J. Cahn and G. Kalonji in [6]. Finally, the model was applied to a larger simulation of crystalline grain growth.

The main advantage of our approach is the possibility to easily adjust the parameters in the equations to given anisotropic surface energies and mobilities. The relation between the GinzburgLandau free energy and the surface energy is non-trivial. This is due to the fact that the stationary wave solution needed in (2.7) is in general not explicitely known. Our choice of an obstacle potential made it possible to find a simple relation between parameters in the multi-phase field system and the sharp interface limit (see [14] for more details).

It turned out that our choice of the free energy which contained a gradient term depending on $\mathbf{u}$ gives enough freedom to choose different anisotropies for interfaces belonging to different indices $i$ and $j$. To our knowledge no other phase field model has been shown to have the same property.

\section{Appendix}

Here, we compute the Wulff shape which corresponds to the crystalline anisotropies, which we introduced in Section 2. Since the result is not restricted to the planar case, we formulate it in $d$-dimensional space.

Let $\eta^{1}, \ldots, \eta^{n} \in \mathbb{R}^{d} \backslash\{0\}$, be such that

$$
\sigma(v):=\max \left\{v \cdot \eta^{k} ; k=1, \ldots, n\right\}
$$

is positive for all $v \in \mathbb{R}^{d} \backslash\{0\}$. This requirement is equivalent to the fact that the intersection of the half spaces

$$
\left\{y ; y \cdot \eta^{k} \leqslant 1\right\}
$$

is a bounded set. The Wulff shape $\mathcal{W}$ to a surface energy $\sigma$ is given by

$$
\mathcal{W}=\left\{x \in \mathbb{R}^{d} \mid x \cdot y \leqslant \sigma(y) \text { for all } y \in \mathbb{R}^{d}\right\}
$$

We now demonstrate that the Wulff shape has $\eta^{1}, \ldots, \eta^{n}$ as its vertices.

THEOREM 1 The Wulff shape $\mathcal{W}$ to a surface energy $\sigma$ defined in (A.1) is the convex hull of $\eta^{1}, \ldots, \eta^{n}$.

Proof. Let us assume that $x$ lies in the convex hull of $\eta^{1}, \ldots, \eta^{n}$. Then there exist $\alpha^{k} \in[0,1]$, $k=1, \ldots, n$, with $\sum_{k=1}^{n} \alpha^{k}=1$ such that

$$
x=\sum_{k=1}^{n} \alpha^{k} \eta^{k} .
$$


For all $y \in \mathbb{R}^{d}$ it holds

$$
x \cdot y=\sum_{k=1}^{n} \alpha^{k} \eta^{k} \cdot y \leqslant \sum_{k=1}^{n} \alpha^{k} \sigma(y)=\sigma(y)
$$

which implies that $x$ belongs to the Wulff shape $\mathcal{W}$.

It remains to show that $\mathcal{W}$ is a subset of the convex hull of $\eta^{1}, \ldots, \eta^{n}$. Since $\mathcal{W}$ is convex, it is enough to show that the points $\eta^{1}, \ldots, \eta^{n}$ belong to the Wulff shape. We can conclude that $\eta^{k} \in \mathcal{W}$ because

$$
\eta^{k} \cdot y \leqslant \max _{l=1, \ldots, n} y \cdot \eta^{l}=\sigma(y)
$$

for all $y \in \mathbb{R}^{d}$. This proves the theorem.

\section{Acknowledgments}

Our work was supported by the German Research Foundation (DFG) through the SFB256 Nichtlineare partielle Differentialgleichungen, and through the grants SA335/25-2 and SA335/25-3 Kurzzeitmetallurgie.

\section{REFERENCES}

1. Angenent, S. \& Gurtin, M. Multiphase thermodynamics with interfacial structure 2. Evolution of an isothermal interface. Arch. Rat. Mech. Anal. 108, (1989) 323-391.

2. Belletini, G., Goglione, R., \& Novaga, M. Approximation to driven motion by crystalline curvature in two dimensions. Adv. Math. Sci. Appl. (to appear).

3. Blowey, J. F. \& Elliott, C. M. Curvature dependent phase boundary motion and parabolic obstacle problems. IMA Volumes in Mathematics and its Applications 47, (1993), Springer-Verlag, New York, pp. 19-60.

4. BRONSARD, L. \& REITICH, F. On three-phase boundary motion and the singular limit of a vectorvalued Ginzburg-Landau equation. Arch. Rat. Mech. Anal. 124, (1993) 355-379.

5. CAHn, J. W. Critical point wetting. J. Chem. Phys. 8, 66, (1977) 3667-3672.

6. CAhn, J. W. \& Kalonji, G. Symmetries of grain boundary trijunctions. J. Phys. Chem. Solids 55, 1994) 1017-1022.

7. De Mottoni, P. \& Schatzman, M. Geometrical evolution of developed interfaces. Trans. AMS 347, (1995) 1533-1589.

8. Elliott, C. M. Approximations of curvature dependent interface motion, University of Sussex CMAIA Research report 96-21 (1996). State of the Art in Numerical Analysis, IMA Conference Proceedings Vol. 63, pp. 407-440. Clarendon Press, Oxford (1997).

9. Elliott, C. M. \& Luckhaus, St. A generalized diffusion equation for phase separation of a multicomponent mixture with interfacial free energy. SFB256 Preprint 195. Bonn (1991).

10. Elliott, C. M. \& Schätzle, R. The limit of the anisotropic double-obstacle Allen-Cahn equation. Proc. Roy. Soc. Edinburgh 126, (1996) 1217-1234.

11. Elliott, C. M. \& Schätzle, R. The limit of the fully anisotropic double-obstacle Allen-Cahn equation in the non-smooth case. SIAM J. Math. Anal. 28, (1997) 274-303.

12. Garcke, H. \& Nestler, B. A mathematical model for grain growth in thin metallic films. Math. Meth. Models Appl. Sci. (to appear).

13. Garcke, H., Nestler, B., \& StOTh, B. On anisotropic order parameter models for multi-phase systems and their sharp interface limits. Physica D 115, (1998) 87-108. 
14. Garcke, H., Nestler, B., \& Stoth, B. A multi-phase-field concept: numerical simulations of moving phase boundaries and multiple junctions, SIAM J. Appl. Math. (in press).

15. Gurtin, M. E. Thermodynamics of Evolving Phase Boundaries in the Plane. Clarendon Press, Oxford (1993).

16. Herring, C. Surface tension as a motivation for sintering. In Kingston, W. E. (ed.), The Physics of Powder Metallurgy, Chapter 8, McGraw-Hill, New York (1951).

17. Hoffmann, D. W. \& CAhn, J. W. A vector thermodynamics for anisotropic surfaces-I. Fundamentals and applications to plane surface junctions. Surface Sci. 31, (1972) 368-388.

18. KIKUCHI, R. \& CAHN, J. W. Theory of interphase and antiphase boundaries in F.C.C. alloys. Acta Met. 27, (1979) 1337-1353.

19. Kobayashi, R. Modelling and numerical simulation of dendritic crystal growth. Physica D 63, (1993) 410-423.

20. Nochetto, R. H., Paolini, M., \& Verdi, C. A dynamic mesh method for curvature dependent evolving interfaces. J. Comput. Phys. 123, (1996) 296-310.

21. McFadden, G. B., Wheeler, A. A., Braun, R. J., Coriell, S. R., \& Sekerka, R. F. Phase field models for anisotropic interfaces. Phys. Rev. E 48, (1993) 2016-2024.

22. Nestler, B. \& WheEler, A. A. Anisotropic multi-phase-field model: Interfaces and junctions. Phys. Rev. E 57, (1998) 2602.

23. Rubinstein, J., Sternberg, P., \& Keller, J. B. Fast reaction, slow diffusion and curve shortening. SIAM J. Appl. Math. 49, (1989) 116-133.

24. Soner, H. M. Motion of a set by the curvature of its boundary. J. Diff. Eqns 101, (1993) 313-372.

25. Soner, H. M. Convergence of the phase-field equations to the Mullins-Sekerka problem with kinetic undercooling. Arch. Rat. Mech. Anal. 131, (1995) 139-197.

26. Steinbach, I., Pezolla, F., Nestler, B., Seesselberg, M., Prieler, R., Schmitz, G. J. \& ReZEnde, J. L. L. A phase-field concept for multi-phase systems. Physica D 94, (1996) 135-147.

27. Sternberg, P. Vector-valued local minimizers of nonconvex variational problems. Rocky Mt. J. Math. 21, (1991) 799-807.

28. Sтотн, B. A sharp interface limit of the phase field equations, one-dimensional and axisymmetric. Eur. J. Appl. Math. 7, (1996) 603-633.

29. TAYLOR, J. E. Constructions and conjectures in crystalline nondifferential geometry. Proceedings of the Conference on Differential Geometry, Rio De Janeiro. Pitman, London (1988).

30. TAYLOR, J. E. Motion of curves by crystalline curvature, including triple-junctions and boundary points. Proceedings of Symposia in Pure Mathematics 54, (1993), 417-438.

31. Thangaraj, N. \& Dahmen, U. Grain boundary structure and morphology in $30^{\circ}\langle 100\rangle$ tricrystal films of Al. MRS Proc. 238, (1992) 171-176.

32. Thangaraj, N., Westmacott, K. H., \& Dahmen, U. Epitaxial growth of (001) Al on (111) Si by vapor deposition. Appl. Phys. Lett. 61, (1992) 913-915.

33. Wheeler, A. A. \& McFadden, G. B. On the notion of a $\xi$-vector and a stress tensor for a general class of anisotropic diffuse interface models. Proc. Roy. Soc. Lond. A 453, (1997) 1611-1630. 